\title{
Space Charge Modelling in Solid Dielectrics under High Electric Field Based on Double Charge Injection Model
}

\author{
G Chen and Su Han Loi \\ School of Electronics and Computer Science \\ University of Southampton, United Kingdom
}

\begin{abstract}
Present study aims to develop a clear insight on factors that influence space charge dynamics in solid dielectrics through a numerical simulation. The model used for the simulation is proposed by Alison and Hill [1] which describes charge dynamics as a result of bipolar transport with single level trapping. In this model, a constant mobility and no detrapping have been assumed. The simulation results show that carrier mobility, trapping coefficient and Schottky barrier have a significant effect on the space charge dynamics. Many features of space charge profiles observed by experiments have been revealed in despite of over simplistic model. More importantly, the simulation allows us to study the role of each individual parameter in the formation of space charge in solid dielectrics, so that the experimental results can be better understood.
\end{abstract}

\section{INTRODUCTION}

One of the major issues in the electricity generation and supply industries is the catastrophic failure of high voltage insulation in power equipment. The problem has received much interest over the last decade as the reliability of power systems has attracted attention of the public. Polymeric materials have been replacing traditional insulating materials due to their excellent mechanical, chemical and electrical properties. However, like any other materials, they are prone to develop an inevitable effect of electrical and chemical degradation after some long-standing operation. It is generally believed that the occurrence of space charge within polymeric materials would introduce an early ageing effect in which the life expectancy of the insulation is greatly affected. Consequently, space charge measurements in polymeric materials have been extensively explored in last two decades. Various mechanisms have been proposed in the process of pursuing understanding observed phenomena. However, majority of the published work concentrates on experimental results and their explanation. Due to the physical and engineering significance of space charge phenomenon, the research on this topic has gradually extended to the numerical-computational technique of analyzing the space charge dynamics via simulation. Various space charge models have been proposed over the years. Earlier models were been extensively reviewed by Lampert and Mark [2] and Mott and Davis [3]. Recently, there have been new developments in charge transportation models following the emerge of new experimental evidences [1,4-5]. Each model has its own merits and drawbacks. Model proposed by Kaneko et al [5] is mainly used to describe the phenomena observed at fields exceeding $100 \mathrm{kV} / \mathrm{mm}$ where so called packet charge is observed. On the other hand, the model proposed by Fukuma et al [4] was based on hopping transport mechanism and its applicability to real system has been hampered 
by instability of the differential equations involved. Recent work by Roy et al [6] revealed that Alison-Hill model [1] shows several features that have been experimentally observed. Transient space charge limited current appears and its peak varies with the applied field is consistent with the observation. More importantly, the reversed current during the depolarisation process has also been revealed.

In this paper we report simulation results based on Alison-Hill model. Several parameters have been investigated to see their influence on charge dynamics and final charge formation in the material. It has been revealed that the experimental result can be matched by simulation.

\section{BASIC GOVERNING EQUATIONS}

Charge transportation in solid dielectrics is essentially governed by a set of basic equations. They describes the behaviour of charge carriers in the system through a time and space dependent total flux $j(x, t)$ and by neglecting diffusion [7]:

Transport equation: $\quad j(x, t)=\mu n(x, t) E(x, t)$

Continuity equation: $\quad \frac{\partial n(x, t)}{\partial t}+\frac{\partial j(x, t)}{\partial x}=s$

Poisson's equation: $\quad \frac{\partial E(x, t)}{\partial x}=\frac{\rho(x, t)}{\varepsilon}$

where $\mu$ is the mobility of carriers, $n$ the density of mobile species, $E$ the electric field, $j$ the current density, $\mathrm{x}$ the spatial coordinate, $\mathrm{t}$ the time, s the source term, $\varepsilon$ the dielectric permittivity and $\rho$ the net charge density.

\section{ALISON-HILL MODEL DESCRIPTION}

The principle on all space charge models lies in the description of the charge conduction and electrical transport mechanism across the material. Alison-Hill model aims to effectively describe the bipolar transport and space charge phenomena in solid dielectrics under high dc stress. The bipolar transport is being described as a conduction process governed by an effective mobility. This feature distinguishes the model from the others. In effect, charge carriers are injected from the electrodes, electrons from the cathode and holes from the anode. Injection occurs based on the Schottky mechanism whereby overcoming a potential barrier at the interfaces. After penetrating into the material, the carriers, under the influence of the applied field, will drift across the material characterized by an effective mobility. Throughout its motions, some carriers are trapped in the localized states i.e. deep trap centres and therefore, the total amount of charges moving across reduces. However, no extraction barrier is introduced in the model and on the other hand, they are prone to recombine with their opposite species (electrons with holes).

Due to the fact that oppositely charged species, electrons and holes are being considered in the numerical computation, charge trapping and mutual annihilation or recombination 
between these species were introduced into the model. The model now contains four species of charge particles namely mobile electrons $\left(e_{\mu}\right)$ and holes $\left(h_{\mu}\right)$, and immobile electrons $\left(e_{t}\right)$ and holes $\left(h_{t}\right)$ (at trap sites) as shown in Figure 1.

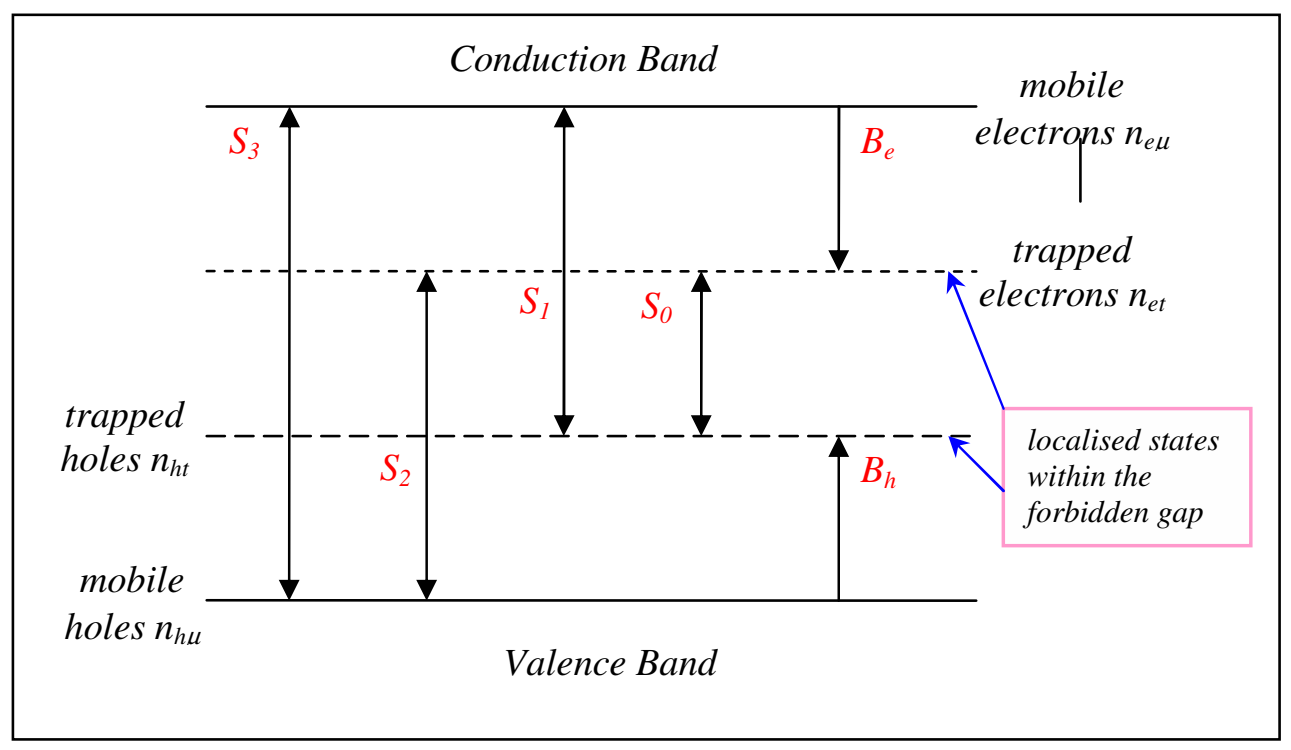

Figure 1 Schematic representation of the conduction and trapping model. $S_{i}$ are recombination coefficients, $\mathrm{n}_{\mathrm{e} \mu}, \mathrm{n}_{\mathrm{et}}, \mathrm{n}_{\mathrm{ht}}, \mathrm{n}_{\mathrm{h} \mu}$ are mobile and trapped electron and hole densities. $\mathrm{B}_{\mathrm{e}}$ and $\mathrm{B}_{\mathrm{h}}$ are electron and hole trapping coefficients.

\section{SIMULATION RESULTS}

Extensive simulation has been carried out by using the computational package MATLAB. The study of space charge phenomenon through numerical modelling analysis is mainly based on the observation made on simulation outcomes. The prediction of the model, which is presented by a graph of charge density against the thickness of the sample, is shown together with graphs of electric field, mobile and trapped electron density and mobile and trapped holes density to form a comprehensive picture of the space charge dynamics overall.

The difference of the present simulation from the others lies in it is experimental oriented. Using the parameters shown in table 1 which are the same as those given in [1], the initial results in Figure 2 give a reasonable description of charge dynamics in the material. Various tests have been carried out to evaluate the significance of each parameter that defines the space charge model. Due to symmetrical behaviour of the model, only the influence of electrons has been extensively studied as their effect in relative to holes is technically similar. Physical parameters that were studied include: trapping coefficient, $\mathrm{B}_{\mathrm{e}}$, mobility of electrons, $\mu_{\mathrm{e}}$, barrier height for the Schottky injection, $\mathrm{w}_{\mathrm{e} i}$, trap density for electron, $\mathrm{E}_{\mathrm{ot}}$ and recombination coefficients, $S_{0}, S_{1}$ and $S_{2}$. Throughout these test, all remaining parameters apart from the one being tested are fixed at their default values as shown in table 1 . Two tests are done on each parameter whereby a higher and a lower to the default value are assigned to the parameter. To reduce significant simulation time 
required, tests are only run to simulate a stressing time up to 600s. However, it has been noticed that charges activity has considerably settled down by then.

\begin{tabular}{|c|c|c|c|}
\hline Parameter & Assigned value & Parameter & Assigned value \\
\hline $\begin{array}{l}\text { Trapping } \\
\text { coefficients }\end{array}$ & & Trap density & \\
\hline $\mathrm{B}_{\mathrm{e}}($ electrons $)$ & $7 \times 10^{-3} s^{-1}$ & $\mathrm{~N}_{0 \mathrm{et}}$ (electrons) & $100 \mathrm{Cm}^{-3}$ \\
\hline $\mathrm{B}_{\mathrm{h}}$ (holes) & $7 \times 10^{-3} s^{-1}$ & $\mathrm{~N}_{0 \mathrm{ht}}($ holes $)$ & $100 \mathrm{Cm}^{-3}$ \\
\hline $\begin{array}{l}\text { Recombination } \\
\text { coefficients }\end{array}$ & & Barrier height for injection & \\
\hline $\mathrm{S}_{0} \mathrm{et}-\mathrm{ht}$ & $4 \times 10^{-3} m^{-3} C^{-1} s^{-1}$ & $\mathrm{w}_{\mathrm{ei}}$ (electrons) & $1.2 \mathrm{eV}$ \\
\hline $\mathrm{S}_{1} \mathrm{e} \mu-\mathrm{ht}$ & $4 \times 10^{-3} m^{-3} C^{-1} s^{-1}$ & $\mathrm{w}_{\mathrm{hi}}($ holes $)$ & $1.2 \mathrm{eV}$ \\
\hline$S_{2} h \mu-$ et & $4 \times 10^{-3} \mathrm{~m}^{-3} \mathrm{C}^{-1} \mathrm{~s}^{-1}$ & Temperature & $300 k$ \\
\hline$S_{3} \mathrm{e} \mu-\mathrm{h} \mu$ & 0 & Applied dc stress, $V$ & $7.5 \mathrm{kV}$ \\
\hline Mobility & & Time per iteration cycle, $\mathrm{dt}$ & $0.01 \mathrm{~s}$ \\
\hline$\mu_{\mathrm{e}}($ electrons $)$ & $9 \times 10^{-15} \mathrm{~m}^{2} \mathrm{~V}^{-1} \mathrm{~s}^{-1}$ & Sample thickness, $d$ & $150 \mu m$ \\
\hline$\mu_{\mathrm{h}}($ holes $)$ & $9 \times 10^{-15} \mathrm{~m}^{2} \mathrm{~V}^{-1} \mathrm{~s}^{-1}$ & Number of divisions, $m$ & 100 \\
\hline
\end{tabular}

Table 1 Definition of parameters controlling dynamic charge interactions
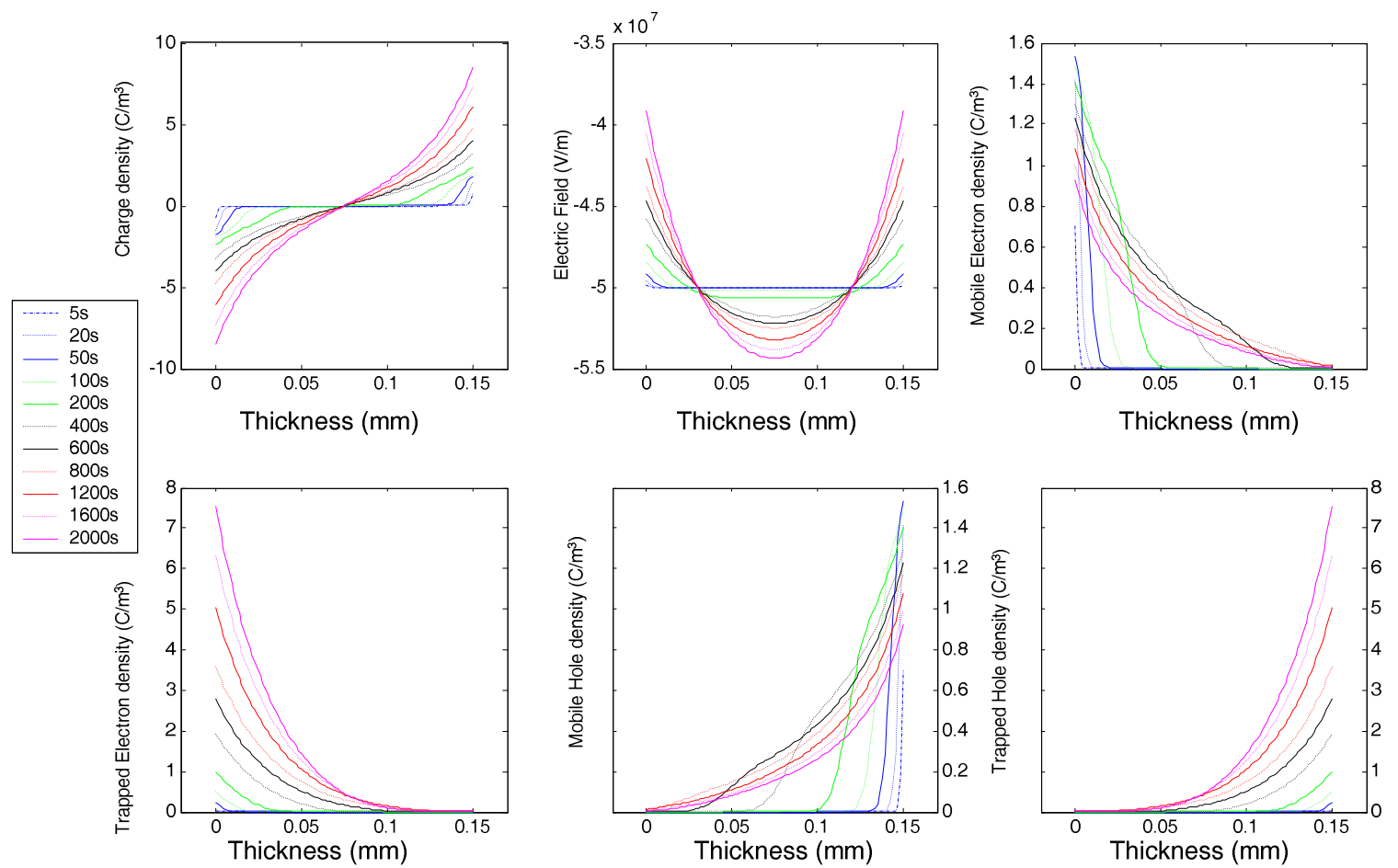

Figure 2 Simulation results based on the parameters given in table 1.

It is clear from the simulation that trapping coefficient, $B_{h}$ and $B_{e}$, mobility of carriers, $\mu_{\mathrm{e}}$ and $\mu_{\mathrm{h}}$, and Schottky barrier, $\mathrm{w}_{\mathrm{ei}}$ and $\mathrm{w}_{\mathrm{hi}}$ are the three dominant parameters that characterize space charge dynamics while $E_{0 t}, S_{1}, S_{2}$, and $S_{3}$, have hardly any noticeable effect on space charge distribution in general. However, a closer look on their individual 
species' dynamics would suggest that these variables would in fact play an important role, to some extent, in defining space charge distributions.

\section{SIMULATION VALIDATION}

Although the simulation results reveal many features of the model, the ultimatum objective is to validate the model with experimental results. To do so, additive-free LDPE film of $150 \mu \mathrm{m}$ thick was stressed at an applied field of $50 \mathrm{kV} / \mathrm{mm}$ for 15 minutes. Space charge distributions in the sample at various times were monitored using the pulsed electroacoustic technique [8]. Figure 3 shows comparison between the simulation and experiment.

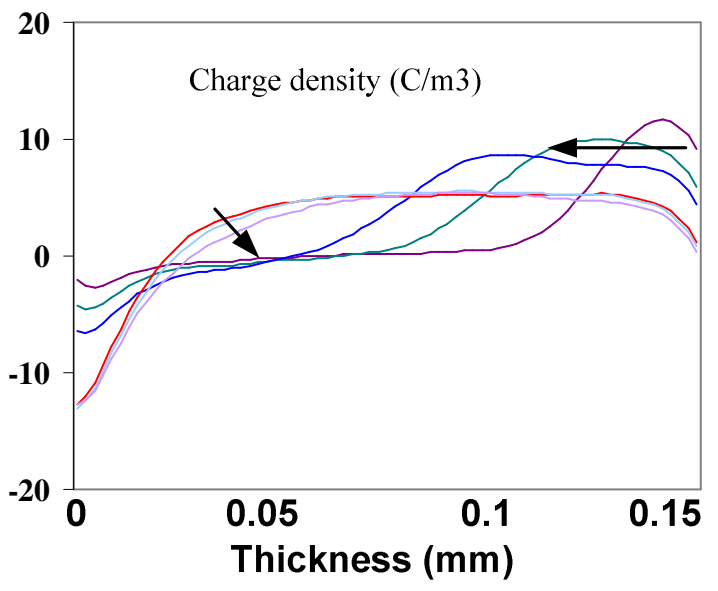

(a) Experimental

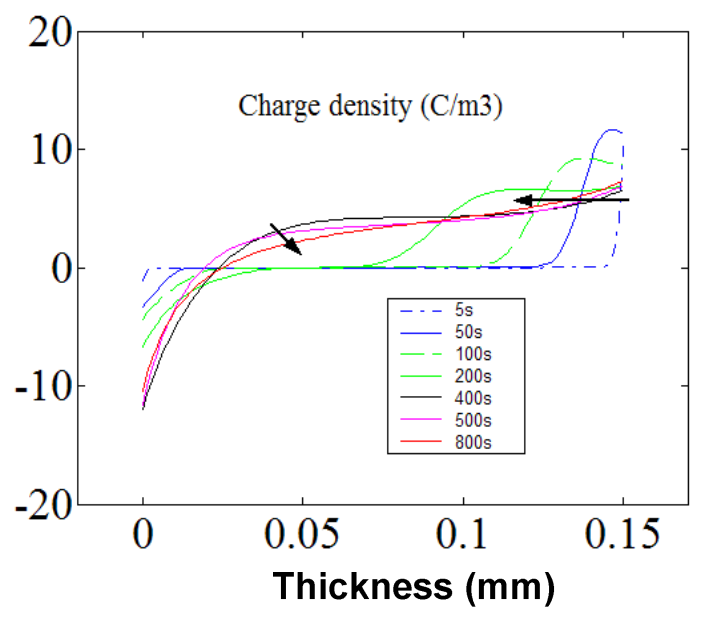

(b) Simulation

Figure 3 Space charge density profiles under an applied filed of $50 \mathrm{kV} / \mathrm{mm}$. simulation (left) and experiment (right)

The simulated charge distributions clearly bear resemblance to the experimental results. A direct comparison of the two graphs suggests a great extent of similarities between the experimental and the computational results. A good match in terms of the charge activities and progression could be observed. Both the plots demonstrate a reasonably close charge settlement point and present also a considerably close match in terms of the magnitude it exhibits. More importantly, once a good match between experiment and simulation has been achieved, both mobile and trapped charges can be separated as shown in Fig. 4. This type of information can not be directly extracted from the experimental results.

\section{SUMMARY}

This paper presents simulated space charge dynamics in solid dielectric materials based on Alison-Hill bipolar charge model. It has been found that the model has satisfactorily represented the behaviour exhibited by the LDPE materials under dc electric field. This numerical simulation approach has enabled the evaluation on the break down of space charge density, which comprises mobile and trapped species of holes and electrons. This allows a clear overview on space charge dynamics to be developed and detail study of 
such area is more approachable. With this, a comprehensive insight on the contribution of each species towards the overall space charge density could be gained. Further work has been undertaken in which the electric field dependent mobility has been considered.
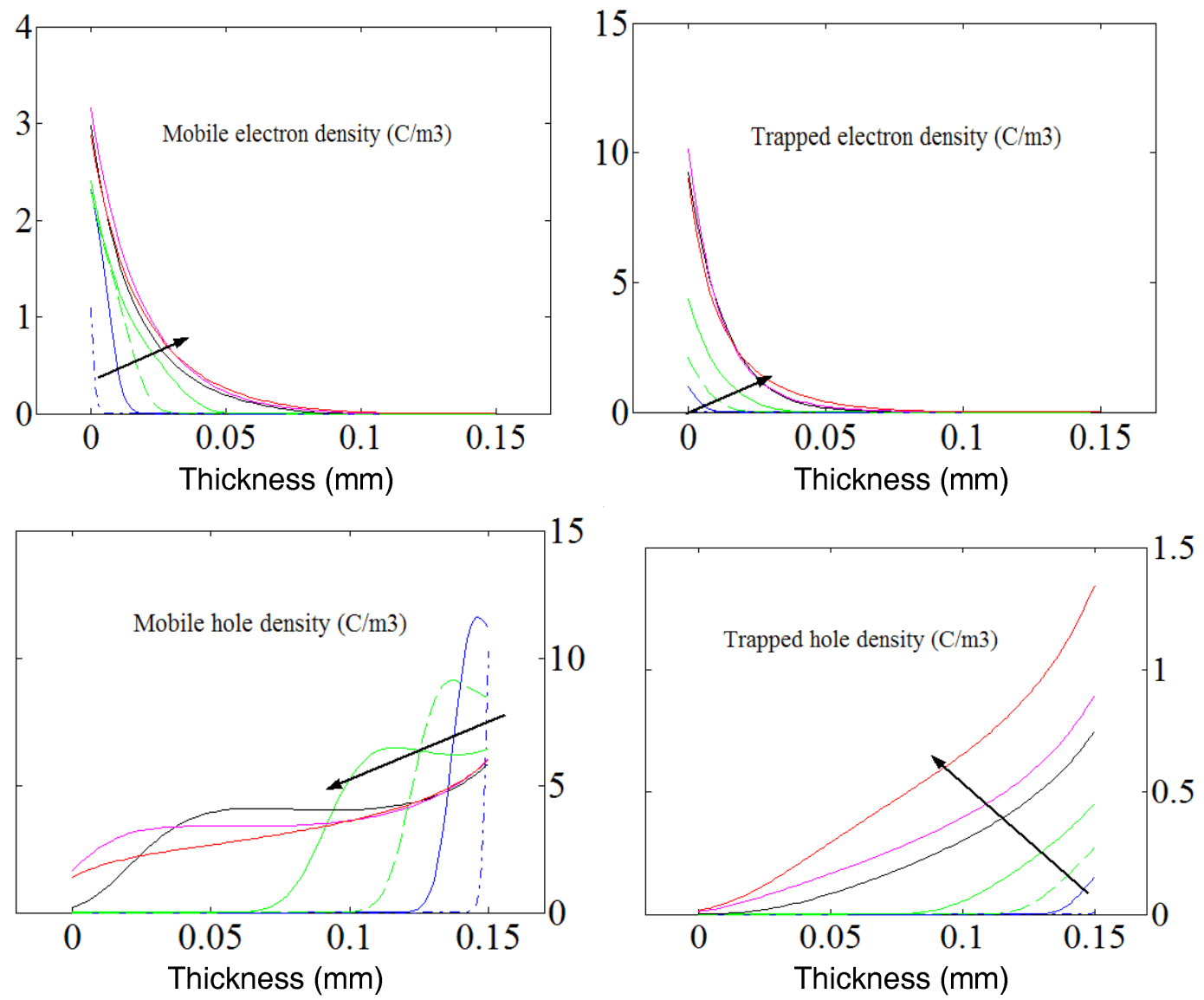

Figure 4 Mobile and trapped charge distributions.

\section{REFERENCES:}

1. J. M. Alison and R. M. Hill J. Phys. D: Appl. Phys. 27 1291-9 (1994).

2. M. A. Lampert and P. Mark, "Current injections in solids", (NY, London, 1970).

3. N. F. Mott and E. A. Davis, "Electronic processes in non-crystalline materials", (Clarendon Press, Oxford, $2^{\text {nd }}$ Ed, 1978).

4. M. Fukuma, M. Nagao and M. Kosaki Proc. 4th Int. Conf.on Properties and Applications of Dielectric Materials (Brisbane) 24-7 (1994)

5. K. Kaneko, Y. Suzuoki and T. Mizutani, IEEE Trans.Dielctr. Electr. Insul. 6 152-8 (1999).

6. S. Le Roy, P. Segur, G. Teyssedre and C. Laurant, J. Phys. D: Appl. Phys. 37 298-305 (2004).

7. K. C. Kao, W. Hwang, "Electrical Transport in Solids", (Pergamon Press, 1981).

8. T. Maeno, T. Futami, H. Kushibe, T. Takada and C. m> Cooke, IEEE Trans. Electr. Insul. 23, 433-439, (1988). 